\title{
BUSINESS OF LIFESTYLE MEDICINE
}

Kriston Kent, MD, MPH

\section{A Unique Lifestyle Medicine Model in the Sunshine State}

From the Editor: One of the biggest challenges in Lifestyle Medicine (LM) is finding a financially viable practice model that is able to deliver high-quality, evidence-based care. LM works; the literature supports these practices. However, less is known about how to be reimbursed appropriately for providing these services in a clinical practice. In this issue, we explore a unique LM model, in Naples, Florida, developed by Kriston Kent, MD, MPH.

\section{Background}

The Kent Center for LIFE, is a small, patient-centered private practice, located in Naples, Florida. The center was opened in 2012 and has remained associated with my current, nonsurgical facial aesthetic practice. I opened this lifestyle medicine (LM) center after 25 years of running a busy aesthetic facial plastic surgery practice. Unlike most LM physicians who originally trained in a primary care specialty, I originally chose a surgical subspecialty for medical practice. A primary reason for my original choice was because I wanted to directly address medical issues, instead of simply treating the symptoms.
However, in 2010, I realized that the budding specialty of LM offered the opportunity to address the root causes of most diseases as opposed to simply slowing down the progression. This inspired me to obtain formal education and training in the field of LM. First, I obtained a master's in public health (MPH) in lifestyle medicine from Loma Linda University. Then I acquired clinical disease processes, like gout, arthritis, and diverticulitis, also exist in some of my patients, and these diseases have responded well to our treatment plans too. Since I still maintain a nonsurgical facial plastic surgery practice, I typically see patients in the Kent Center for LIFE 3 days per week. A typical half-day in the LM clinic usually consists of 3 new or return patient encounters.

\section{The Kent Center for LIFE, is a small, patient-centered private practice, located in Naples, Florida.}

skills in LM behavior modification at the Institute of Lifestyle Medicine at Harvard and at the St. Helena Center for Health in Napa Valley, California.

\section{Introduction}

My current practice focuses on helping patients prevent and/or reverse chronic disease processes including metabolic syndrome, type 2 diabetes, hypertension, hyperlipidemia, dementia, some cancers, and cardiovascular disease. Several other

\section{Practice Demographics}

Naples tends to be an affluent, relatively healthy coastal town in southwest Florida. It consistently ranks very high in longevity, ${ }^{2}$ has an older than average age demographic, and is currently seeking the designation of a "Blue Zone community" from Healthways. My specific patient population includes a combination of retired individuals who spend part of their time (or all of their time) in Naples,

DOI: 10.1177/1559827616658202. From Kent Center for LIFE, Naples, Florida. Address correspondence to Kriston Kent, MD, MPH, Kent Center for LIFE, 840111 th Avenue North, Suite 9, Naples, FL 34108; E-mail: kkent@tkclife.com.

For reprints and permissions queries, please visit SAGE's Web site at http://www.sagepub.com/journalsPermissions.nav. 
and a group of very busy professionals (workaholics) who are highly successful in their professional life, but are pending disasters from a health perspective. Most of them are already on multiple medications for a myriad of chronic diseases. My patients tend to be evenly split between men and women, and I do not currently treat children.

\section{The Process}

We initially consult (visit 1) with the patient to identify their concerns and their appropriateness for LM treatment. Next, we perform an extensive assessment (visit 2) and evaluation (visit 3) of the patient's current health status and health habits. Once the challenges are identified, we primarily focus on changing lifestyle habits (visit 4+). This usually leads to reducing or eliminating medications and improving quality of life.

\section{Visit 1: The Consultation Visit}

The initial patient interaction involves a 1- or 2-hour consultation session. If the patient wishes to proceed, and I believe that we can address the patients' problem(s), 2 options are then offered. One option is the Advanced Initial Assessment (AIA), which includes nutrition and physical activity evaluations, a healthy habits score, biometrics, a modified Mediterranean diet score, and a rather extensive laboratory evaluation. The second option, the Advanced Initial Team Assessment (AITA) option, includes all of the above, but also includes a teamoriented approach utilizing health care members who see patients at the Kent Center on request. The team-oriented approach includes 1-hour evaluations by each of our team members, including a licensed clinical dietitian/nutritionist, a physical therapist, and the behaviorist.

\section{Visit 2: The Advanced Initial Assessment-Data Gathering}

Irrespective of the Initial assessment option a patient chooses (AIA or AITA), a second appointment is scheduled to perform an assessment. At this data-gathering visit, specific biometrics evaluated include height, weight, body mass index, body composition (using bioelectrical impedance), waist circumference, pulse, and blood pressure. Laboratory evaluation includes an advanced lipid profile, an inflammatory profile, a metabolic profile, a limited genetic profile, and a micronutrient profile. We draw the blood for the patient in our office. Our modified Mediterranean Diet Score includes evaluation of daily/ weekly intake of fruits, vegetables, whole grains, beans/legumes, nuts, olive oil, omega-3 fats, red meat, poultry, and dairy. In general, points are gained by increasing amounts of the first 7 food groups and decreasing amounts of the last 3. Our Healthy Habits Score includes evaluation of self-awareness, quality of diet, amount of water consumption, amount of physical activity, quality of sleep, deep breathing of fresh air, daily sun exposure, quality down time, presence of addictions, positive/negative thinking, spirituality, and knowledge of key health indicators.

\section{Visit 3: The Advanced Initial Assessment Report- Data Discussion}

Prior to the third visit, I (the LM physician) review all of the data gathered from the initial assessment (AIA or AITA) and generate a comprehensive report. This report is extensively reviewed with the patient during the in-office visit. In the summary portion of this Advanced Initial Assessment Report, the patient's strengths and challenges are highlighted. At the end of the report, specific recommendations are made on how to address the challenges that have been identified. If the initial assessment is an AITA, the report includes assessment and recommendations from the other team professionals in addition to assessment and recommendations from the LM physician. The patient is then offered an opportunity to spend either 6 or 12 months with their LM physician serving as their physician/health coach to execute their specific behavior modification plan and achieve their health goals.
Visit 4+: Lifestyle Medicine Plans to Address the Cause

More specifically, patients are offered 4 different LM care packages: Silver, Gold, Platinum, and Diamond.

The basic plans (there are 2: Silver and Gold) include a 6-month or a 1-year plan. Either way, the patient has scheduled visits to meet with the LM physician and staff 1 or 2 times per month. Additional visits are available at no additional charge.

The simplest plan is the Silver package. Its duration is 6 months and it includes at least monthly visits with the LM physician and his staff as well as free admission to the monthly healthy habits lectures.

The other basic plan is our Gold package. Its duration is 12 months and it includes monthly visits with the LM physician and his staff as well as free admission to the monthly healthy habits lectures.

In addition to the basic plans noted above, there are 2 premium plan options. The premium plans allow patients to have additional visits with multiple providers (eg, nutritionist, physical therapy/exercise specialist, behaviorist) throughout the year (in addition to the LM physician).

The Platinum package is a 12-month plan that includes meetings with 2 out of the 3 of our other health care team professionals (eg, nutritionist, physical therapist, behaviorist/health coach).

Finally, the Diamond package provides 12 months of meetings with a LM physician and a specified number of meetings with our nutritionist, our physical therapist, and our behaviorist/ health coach.

Regardless of the package chosen, throughout the 6- or 12-month process, biometrics are evaluated at each visit and healthy habits scores and diet scores are evaluated at least quarterly. Laboratory testing is repeated as indicated, but is at least retested yearly. No limits are set on the number of visits with the LM physician or patient to physician/office staff contacts. A defined number of visits are specified when the patient desires additional meetings with the nutritionist, the physical therapist, and/or the behaviorist/health coach. 
After completion of the 6- or 12-month package, most patients feel confident and comfortable continuing their lifestyle changes. A minority of patients, approximately $25 \%$, will sign up for an additional package. Regardless, patients are always welcome to return for a reassessment.

\section{Additional Services Offered}

A series of monthly healthy habits lectures are also provided throughout the year to teach our twelve healthy habits more in-depth, and to provide the benefits of patient-to-patient interaction. Handouts are provided at each presentation. For selected postmenopausal women, and older men, salivary hormone levels are tested, including testing for levels of estrone, estradiol, estriol, progesterone, DHEA (dehydroepiandrosterone), and testosterone. Salivary cortisol levels at awakening, lunch, dinner, and bedtime are also measured in some patients.

In addition to the LM services, I still offer an array of facial aesthetic treatment options in my practice and these include injectable fillers, neuromuscular relaxing agents, and cytokine-rich plasma treatments for facial, neck, and décolleté skin aging. Cytokine-rich plasma is used to improve androgen-induced alopecia in selected men and postmenopausal women.

\section{Finances}

With respect to financial reimbursement, the services of my practice are provided to patients via a self-pay model.

The consultation visit has a separate fee. The Advanced Initial Assessment visit and the Advanced Initial Assessment Report visit have a separate single fee. Most of the laboratory work is covered by insurance.

Finally, each 6- or 12-month package has a comprehensive fee. This covers the cost of all of the visits and services offered in the chosen package.

\section{Summary}

In summary, The Kent Center for LIFE is a self-pay, patient-centered, LM model that utilizes a team approach to deliver high-quality care. It benefits from my previously established practice and reputation, as well as its location in a relatively affluent area. Providing long-term LM package options, with essentially unlimited visits, affords us the opportunity to take the time needed to help patients make complex lifestyle changes. With the Kent Center for LIFE team's help, guidance, and encouragement, many patients achieve better management of their chronic diseases, change their lives in ways they never thought possible, and discover what true health really is. AJLM

\section{References}

1. Kent Center for LIFE. Welcome page. http://www.tkclife.com. Accessed April 1, 2016.

2. County health rankings and roadmaps. Princeton, NJ: A Robert Wood Johnson Foundation Program. http://www. countyhealthrankings.org/app/ florida/2016/rankings/collier/county/ outcomes/overall/snapshot. Accessed April 1, 2016. 